Noname manuscript No.

(will be inserted by the editor)

\title{
Redundant Disequalities in the Latin Square Problem
}

\author{
Bart Demoen - Maria Garcia de la Banda
}

Received: date / Accepted: date

\begin{abstract}
This paper studies the redundancy properties of the constraints used when formulating the well known Latin Square problem. This problem is often formulated using either $(N-1) * N^{2}$ binary disequalities or $2 * N$ all_different global constraints. Both formulations contain redundant constraints. A complete classification of all redundant sets of constraints, be they binary or global, is performed for any $N$.
\end{abstract}

Keywords Constraint Programming · Redundant constraints

\section{Introduction}

The search for a solution to a constraint problem can sometimes be sped up by either adding or removing redundant constraints (also called implied or entailed constraints; see for instance pages 376 and 431 in [8]), that is, constraints that do not change the set of solutions to the problem. Therefore, it is useful to be able to identify redundant constraints in a problem specification (or model), and also to infer new, redundant constraints that could be added to it. Redundant and implied constraints, and their effects on performance, have been studied for instance in [10] and [5] in the context of Linear Programming, and in [1] and [7] in Constraint Programming. In particular, [7] studied the

This research was partly sponsored by he Australian Research Council grant DP110102258, and by the Research Foundation Flanders (FWO) through projects WOG: Declarative Methods in Computer Science and G.0221.07

B. Demoen

Dept. of Computer Science, KU Leuven, Belgium

Tel.: +32-16-327547

Fax: $+32-16-327996$

E-mail: Bart.Demoen@cs.kuleuven.be

M. Garcia de la Banda

Caulfield School of IT, Monash University, VIC 3145, Australia 
addition of constraints to the usual definition of the cardinality matrix problem (which includes the Latin Square) to improve its performance. Our motivation is however different: our aim is to study the redundancy properties of the constraints already present in the problem, rather than infer new redundant constraints.

While disequalities are commonly used to model constraint problems, determining whether a disequality is redundant or not is not easy due to, among other things, their lack of transitivity and their dependence on the domains of the variables. For instance, from a chain of disequalities $x_{1} \neq x_{2} \neq \cdots \neq x_{n}$ between boolean variables, one may conclude that $x_{1} \neq x_{4}$ (amongst others), but if the domains have a larger cardinality, this conclusion is no longer valid.

This paper studies the redundancy properties of the disequality constraints used for modelling the Latin Square [2] problem of size $N$, which requires filling out an $N^{2}$ square with numbers from 1 to $N$ in such a way that each row and column contains every number exactly once. A common model of Latin Square as a constraint satisfaction problem uses (a) $N \times N$ variables $x_{i j}, i, j \in[1 . . N]$, representing the value assigned to the cell in row $i$ and column $j$ of the square, (b) $N^{2}$ domain constraints indicating that the domain of each variable $x_{i j}$ is [1..N], and (c) $2 N$ all_different global constraints [6,4] of $N$ variables each, one for the variables in each column and in each row. We refer to this model, that is, to the set of variables, domain constraints and all_different constraints, as LatinSquare $(N)$.

An alternative model substitutes each of the all_different global constraints by the conjunction of the $N *(N-1) / 2$ binary disequality constraints on its input variables. For example, all_different $(\{x, y, z\})$ is substituted by the conjunction of constraints $x \neq y, x \neq z$, and $y \neq z$. We refer to this alternative model as LatinSquareBin $(N)$. It has the same number of variables and domain constraints as LatinSquare $(N)$, but $N^{2} *(N-1)$ disequalities rather than $2 N$ all_different global constraints.

We provide a complete classification of all redundant and non-redundant sets of constraints used in LatinSquare $(N)$ and LatinSquareBin $(N)$. To achieve this, we first classify any pair of disequalities as either redundant or non-redundant. This works well for Latin Square because the number of different pairs of disequalities is small when considered up to symmetry, that is, when considered up to the spatial symmetries of a square (any row, column and diagonal symmetries). Once this is done, we prove that any set of disequalities either contains at least one non-redundant pair and is, therefore, non-redundant, or otherwise is redundant. We believe this method will work also for other problems in which a limited number of small patterns of disequalities provably occurs in any non-redundant set of disequalities. Constraint satisfaction problems with many symmetries seem good candidates. While our proofs are formal, some of the intuition behind the proofs resulted from running a constraint logic program for small values of $N$. We used B-Prolog [11] for this, although any Prolog with constraints would be adequate. 


\section{Redundancy of sets of binary disequalities in LatinSquareBin(N)}

Our results for disequalities can be easily visualized using a constraint graph where nodes represent the variables in LatinSquareBin $(N)$ and edges represent the disequalities. We define two particular subsets of these edges:

$$
\begin{aligned}
& R_{1}(N)=\left\{\left(x_{1 i}, x_{1 j}\right) \mid 1 \leq i<j \leq N\right\} \\
& R_{2}(N)=\left\{\left(x_{11}, x_{1 j}\right) \mid 1<j \leq N\right\} \cup\left\{\left(x_{i 1}, x_{j 1}\right) \mid 1<i<j \leq N\right\}
\end{aligned}
$$

Informally, $R_{1}(N)$ contains all disequalities between any two variables in row 1 , while $R_{2}(N)$ contains all disequalities in row 1 involving the top-left variable $\left(x_{11}\right)$, and also all the disequalities in column 1 not involving $x_{11}$. Figure 1 shows these sets for $N=4$.

This section proves not only that both $R_{1}(N)$ and $R_{2}(N)$ are redundant sets of dise-

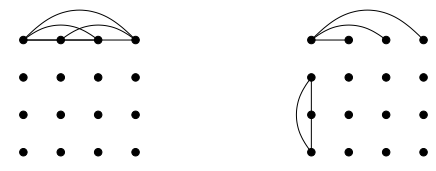

Fig. 1: $R_{1}(4)$ and $R_{2}(4)$ qualities, but also that, up to symmetry, they are the only maximal redundant sets. That is, the only ones that cannot be extended without becoming non-redundant. Note that when we say "up to symmetry" we refer to the symmetries of the square, that is, those formed by exchanging any two rows, any two columns, reflecting the square upon any diagonal, or any combination of these. While Latin Square has other symmetries (e.g., those formed by exchanging any two values), these are irrelevant, since the symmetries we are considering are those of the associated constraint graph only, not all those of the Latin Square problem.

$2.1 R_{1}(N)$ and $R_{2}(N)$ are redundant sets of disequalities

Lemma 1 For all $N>0, R_{1}(N)$ is a redundant set of disequalities.

Proof Consider the model obtained from LatinSquareBin $(N)$ by removing the disequalities in $R_{1}(N)$. Let $s$ be any solution of this modified model, and $I$ any number in $[1 . . N]$. Since in every column of $s$ all elements are known to be different, $s$ must contain $N$ occurrences of the number $I$, one per column. Since in every row, except the top one, all elements are also known to be different, the lower rows must contain $N-1$ occurrences of $I$. Thus, the remaining occurrence of $I$ must be in the top row. Since this reasoning applies to each value in $[1 . . N]$, the top row must contain every $I \in[1 . . N]$. As a result, all variables in the first row of any solution $s$ must be different and, therefore, $R_{1}(N)$ is redundant.

Lemma 2 For all $N>0, R_{2}(N)$ is a redundant set of disequalities.

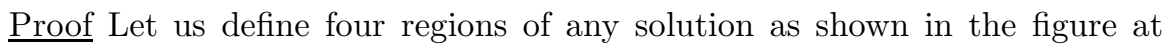


the right: $\mathrm{A}$ as the upper left cell, B the upper row without $\mathrm{A}, \mathrm{C}$ the left column without $\mathrm{A}$, and $\mathrm{D}$ the rest of the square. Consider the model obtained by removing from LatinSquareBin $(N)$ the disequalities in $R_{2}(N)$. It is easy to see that for any solution $s$ and any number $I$ in region $B$ of $s$, the number of occurrences of $I$ in $B$ is 1 , since there are disequalities between each pair of variables in $B$. Also, $I$ occurs $N-1$ times in $B \cup D$, since there are disequalities between each pair of variables in

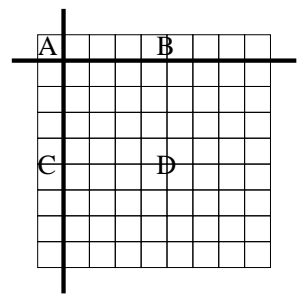
any of its columns. Therefore, $I$ occurs $N-2$ times in $D$. Further, $I$ also occurs $N-1$ times in $C \cup D$, since there are disequalities between each pair of variables in any of its rows. Therefore, $I$ occurs once in $C$.

As a result, the set of numbers occurring in $C$ and occurring in $B$ are the same, which means all_different $(C)$ holds for any $s$. Further, since the number in $A$ cannot appear in $B$, all disequalities in $R_{2}(N)$ hold for any $s$ and, therefore, $R_{2}(N)$ is redundant.

Let $R(N)=\left\{R_{1}(N), R_{2}(N)\right\}$ and $S$ denote any set of disequalities. We say that $S$ is covered by $R(N)$ if, up to symmetry, $S$ is a (possibly non-strict) subset of $R_{1}(N)$ or $R_{2}(N)$. Otherwise, we say $S$ is not covered. It is clear that any set $S$ covered by $R(N)$ is redundant.

\subsection{Non-redundant pairs of disequalities}

Clearly, any single disequality is covered by $R(N)$. We thus turn our attention to pairs of disequalities. Figure 2 shows all pairs of disequalities in LatinSquareBin(4) up to symmetry. We denote them by $P_{i}, i=1 . .8$ as numbered in the figure. For $N>4$, the exact same eight pairs exist, and up to symmetry, no more. This can be proven by exhaustively considering all possibilities as follows: two different disequalities have a variable in common or not. In the former case, the three variables lie either in the same row $\left(P_{7}\right)$ or not $\left(P_{4}\right)$. In the latter case, the four variables lie either in the same row $\left(P_{8}\right)$, in two different rows $\left(P_{1}, P_{2}\right.$ and $\left.P_{3}\right)$, or in three different rows $\left(P_{5}\right.$ and $\left.P_{6}\right)$.
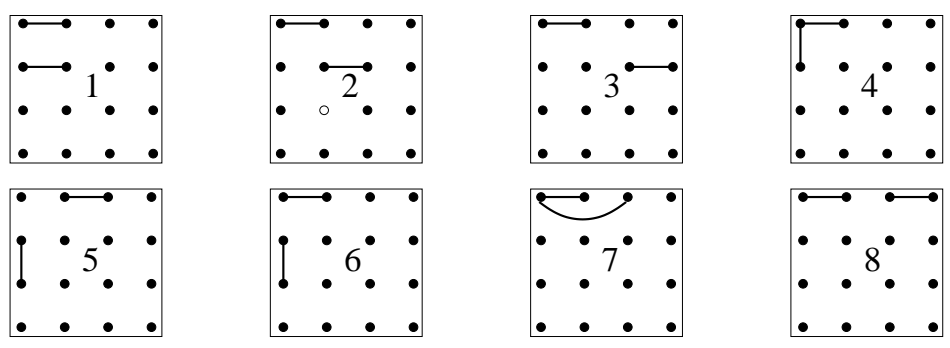

Fig. 2: All possible pairs of disequalities for $N=4$, up to symmetry

For $N=3, P_{3}$ and $P_{8}$ do not exist, and for $N=2$, only $P_{1}$ and $P_{4}$ exist. 
Clearly, pairs $P_{6}, P_{7}$ and $P_{8}$ are covered by $R(N)$ and, therefore, they are redundant. None of the remaining pairs is covered by $R(N)$ and, as we prove, they are all non-redundant.

Theorem 1 Consider the model obtained by removing from LatinSquareBin(N) the disequalities in some $P_{i}, i \in 1 . .5$. For $N>3$, this modified model has at least one more solution than LatinSquareBin(N).

Proof We first prove the case $N=4$ : we present graphical proofs for each $P_{i}$ in the style of [3], where each proof consists of a box with two pictures. The top picture represents a solution to LatinSquareBin(4) where only a few numbers are explicitly given (it is easy to see that each top picture can be completed to several solutions). The bottom picture is obtained from the top one by altering only the encircled numbers, and it represents a solution to the model obtained by eliminating $P_{i}$, that is not a solution to LatinSquareBin(4) (as shown by the violated disequalities displayed in the picture as an arc between two numbers). Together, the two pictures of box $i$ show that there exists a solution to the model obtained by eliminating $P_{i}$, that is not a solution of LatinSquareBin(4).
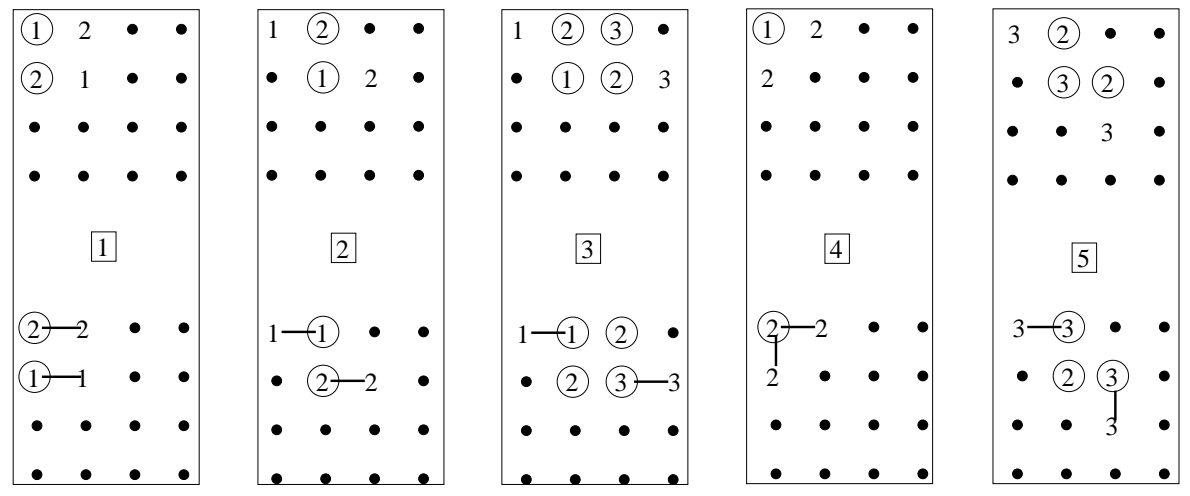

While the pictures above give a proof for $N=4$, it is straightforward to see that they apply to any $N>3$, since one can simply add more dots for extra columns and rows. One merely needs to prove that there is always a solution of LatinSquareBin(N) with $N>3$ starting with the numbers explicitly given in the top picture of each box. This can be done easily for boxes 2 up to 5 by rotating the successive rows. For box 1, one can use Theorem 2 from [9].

2.3 Up to symmetry, $R_{1}(N)$ and $R_{2}(N)$ are the only maximal redundant sets

Theorem 2 Every set $S$ of disequalities from LatinSquareBin(N) with $N>3$ either contains a non-redundant pair or is covered by $R(N)$.

Proof There are three cases for $S$. (1) All disequalities in $S$ affect variables in the same line (i.e., column or row). In this case $S$ is clearly covered by $R_{1}(N)$ and does not contain a non-redundant pair. (2) Some disequalities of $S$ appear 
in different parallel lines. In this case, $S$ must contain a non-redundant pair from either $P_{1}, P_{2}$ or $P_{3}$, and is thus not covered by $R(N)$. (3) All disequalities of $S$ must now appear in exactly two crossing lines. Let us assume, without loss of generality, that the lines are column 1 and row 1 . By Figure 2 we know that any crossing pair in $S$ must be in $P_{4}, P_{5}$ or $P_{6}$. If $S$ contains a pair in $P_{4}$ or $P_{5}$, then $S$ contains a non-redundant pair and is not covered by $R(N)$. Else, $S$ is only allowed to have pairs of disequalities derived (via symmetry) from $P_{6}$ and, thus, they are all in $R_{2}(N)$.

It follows from the above theorem that $R_{1}(N)$ and $R_{2}(N)$ are the only two maximal redundant sets of disequalities for $N>3$.

The special case $N=3$ : The reasoning used in Theorem 1 to prove that $P_{1}$ is a non-redundant pair is not valid for $N=3$, since the initial configuration used in box 1 cannot be completed to a solution of LatinSquareBin(3). However, for $N=3$ it is easy to exhaustively enumerate all maximal sets of disequalities, which are shown in Figure 3. Note that the rightmost set contains $P_{1}$ and therefore (by Theorem 1) cannot be generalized to $N>3$.

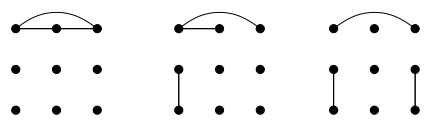

Fig. 3: All maximal redundant sets of disequalities for $N=3$

\section{Redundancy of all_different constraints in LatinSquare(N)}

Theorem 2 directly implies the following result:

Corollary 1 Exactly one all_different constraint in the formulation of LatinSquare $(\mathrm{N})$ is redundant.

Proof Every single all_different constraint is clearly redundant, as every such constraint is equal to $R_{1}(N)$ up to symmetry and, thus, covered by $R(N)$. Moreover, no two all_different constraints together can be covered by $R(N)$, since they always would contain a non-redundant pair: either in $P_{1}, P_{2}$ and $P_{3}$ if the constraints appear in parallel lines, or in $P_{4}$ and $P_{5}$ if they cross. Therefore, no combination of two all_different constraints is redundant.

\section{Conclusion}

This paper studies the redundancy relationships of the binary disequality constraints and global all_different constraints used to model the Latin Square program of size $N$. In particular, we have identified all maximal redundant sets found both when the problem is specified using all_different constraints, and when it is specified using binary disequality constraints. For the former case, 
we have proved that every all_different constraint is redundant and no two such constraints are redundant. For the latter case, we have characterized all possible sets of binary equalities as either covered by one of our two maximal redundant sets, or containing a non-redundant pair. That is, a pair of disequalities that - if missing - allows the problem to have solutions other than those of LatinSquare $(N)$. We find the fact that a single pair of disequalities can modify the problem so fundamentally, quite surprising. A small experiment showed that taking out the redundant constraints from the problem description reduces performance by an order of magnitude even for problem sizes as small as $N=6$, and that this reduction grows with $N$.

The method used to prove the above results is based on first classifying any pair of disequalities $\left(P_{1}, \cdots, P_{8}\right)$ as either redundant or non-redundant, and then proving that any set of disequalities $S$ either contains at least one non-redundant pair and is, therefore, non-redundant, or otherwise is redundant. We believe this method will work also for other problems in which a limited number of small patterns of disequalities provably occurs in any nonredundant set of disequalities. Note that both the results and the methodology are quite different from those in [3], where we proved for $3 \times 3$-Sudoku that up to six all_different constraints (out of the usual 27 with which the problem is defined) are redundant, and we gave a full classification of such sets of six. We also found that many subsets of 162 disequalities are redundant for $3 \times 3$ Sudoku and conjectured that no more is possible. No results for NxN-Sudoku for general $N$ were obtained. In contrast, for the Latin Squares problem, a full classification is obtained not only for the redundant all_different constraints, but also for the redundant binary disequality constraints. Moreover, the results were obtained for all problem sizes $N$. Further, the methodology used in [3] was based on a series of constructive lemmas where given a set of disequalities, a new redundant disequality was inferred.

We hope that by studying particular constraint problems (like Latin Square here, and Sudoku elsewhere [3]), we will get a varied toolset of methods with which to determine the redundancy properties of disequality constraints in a wide set of problems.

Acknowledgements We thank Ian Wanless for pointing us to relevant literature on Latin Squares, and anonymous reviewers for suggestions that improved the presentation.

\section{References}

1. Bessière C, Coletta R, Petit T (2007) Learning implied global constraints. In: Veloso MM (ed) International Joint Conference on Artificial Intelligence, pp 44-49

2. Colbourn C, Dinitz J, Wanless I (2007) Latin squares. In: Colbourn C, Dinitz J (eds) The CRC Handbook of Combinatorial Designs, Chapman\&Hall/CRC, Boca Raton, pp 135-152 
3. Demoen B, Garcia de la Banda M (2013) Redundant Sudoku Rules. To be published in the Journal of Theory and Practice of Logic Programming

4. Gent IP, Miguel I, Nightingale P (2008) Generalised arc consistency for the alldifferent constraint: An empirical survey. Artif Intell 172(18):1973-2000, DOI 10.1016/j.artint.2008.10.006, URL http://dx.doi.org/10.1016/j.artint.2008.10.006

5. Greenberg HJ (1996) Consistency, redundancy, and implied equalities in linear systems. Annals of Mathematics and Artificial Intelligence 17(1):3783

6. van Hoeve WJ (2001) The alldifferent constraint: A survey. URL http://www.citebase.org/cgi-bin/citations?id=oai:arXiv.org:cs/0105015

7. Régin JC, Gomes CP (2004) The cardinality matrix constraint. In: Principles and Practice of Constraint Programming-CP 2004, Springer, pp $572-587$

8. Rossi F, van Beek P, Walsh T (eds) (2006) Handbook of Constraint Programming. Elsevier

9. Ryser H (1951) A Combinatorial Theorem with an Application to Latin Rectangles. Proceedings of the American Mathematical Society 2(4):550552

10. Telgen J (1978) Redundant constraints in linear programming problems. Operations Research Verfahren 28:420-433

11. Zhou NF (2012) The language features and architecture of B-Prolog. Theory and Practice of Logic Programming 12(1-2):189-218 